\title{
Ameyali: Evaluación de la usabilidad de la plataforma Integral para el control y monitoreo del servicio de agua potable en comunidades rurales bajo la escala de Sus
}

\author{
A. Montiel-De Jesús ${ }^{1}$, C. Morales-Constantino ${ }^{1}$, S. Ixmatlahua-Díaz ${ }^{1}$ \\ N. Hernández-Chaparro ${ }^{1}$, H. Marín-Vega ${ }^{1}$
}

146wo385@zongolica.tecnm.mx, 146wo386@zongolica.tecnm.mx, sergio.ixmatlahua.pd169@zongolica.tecnm.mx, norma.hernandez.pd183@zongolica.tecnm.mx, humberto_marin_pd177@zongolica.tecnm.mx.

${ }^{1}$ Tecnológico Nacional de México campus Zongolica, km 4 carretera a la compañía S/N, 95005, Zongolica, México.

DOI: 10.17013/risti.36.116-131

\begin{abstract}
Resumen: El internet de las cosas (IoT), es una de las tendencias tecnológicas que marcan un parteaguas en la forma en que controlamos y monitoreamos las actividades de nuestra vida diaria, desde el trabajo hasta el hogar. En ese sentido, en este trabajo, se presentan los resultados obtenidos de la implementación de tecnologías de IoT aplicadas a la mejora de la operación de un sistema de bombeo hidráulico en una comunidad rural. La metodología utilizada es la orientada a Prototipos para obtener un análisis de requerimientos, el diseño de la aplicación mediante diagramas UML y posteriormente el desarrollo de la plataforma integral que incluye una aplicación Web, una aplicación móvil y un módulo electrónico. Los resultados obtenidos muestran que la plataforma desarrollada así como los sensores utilizados son factibles y viables para controlar la bomba del suministro de agua de la comunidad de Huixtitla, Veracruz, ya que favorece la reducción del costo de la automatización del sistema, para el caso de los usuarios facilita el control y monitoreo de manera remota gracias a la aplicación móvil y a nivel nacional es un producto original en su categoría y aplicabilidad.
\end{abstract}

Palabras-clave: Internet de las Cosas; automatización; suministro de agua; ingeniería de software; domótica.

Ameyali: Evaluation of the usability of the Integral platform for the control and monitoring of the drinking water service in rural communities under the scale of Sus

Abstract: The internet of things IoT, is one of the technological trends that mark a watershed in the way we control and monitor the activities of our daily lives, from work to home. In this sense, this paper presents the results obtained from the implementation of IoT technologies applied to the improvement of the operation of a hydraulic pumping system in a rural community. The methodology 
used is Prototype oriented to obtain an analysis of requirements, the design of the application through UML diagrams and later the development of the integral platform that includes a Web application, a mobile application and an electronic module. The results obtained show that the platform developed as well as the sensors used are feasible and viable to control the pump of the water supply of the community of Huixtitla, Veracruz, since it favors the reduction of the cost of the automation of the system, in the case of Users facilitate remote control and monitoring thanks to the mobile application and at the national level it is an original product in its category and applicability.

Keywords: Internet of Things; automation; water supply; software engineering; domotics.

\section{Introducción}

El agua es vital para diversas actividades humanas, en México, el 77\% del agua se utiliza en la agricultura; el $14 \%$ en el abastecimiento público, el 5\% es empleada en las termoeléctricas y un 4\% en la industria por lo que su disponibilidad es un derecho humano y se debe garantizar para toda la población (CONAGUA, 2010).

En el estado de Veracruz, sólo el 44.37\% de las zonas rurales cuentan con servicio de abastecimiento de agua potable (CONAGUA, 2010), una de ellas es el municipio de Soledad Atzompa donde se ubica la comunidad de Huixtitla y a una hora de camino se encuentra el sistema de bombeo que abastece la red de agua a través de tomas en sus domicilios.

Actualmente, la operación del sistema de bombeo del agua en la comunidad de Huixtitla está a cargo de una persona, cuya responsabilidad es encender y apagar diariamente el sistema, además de monitorear su funcionamiento y realizar el mantenimiento. No obstante, la distancia y la orografía del camino son altamente accidentados, poniendo en riesgo la integridad física de la persona a cargo del sistema de bombeo, además de no contar con una administración adecuada de los mantenimientos y servicios que se deben hacer al sistema.

De acuerdo con lo anterior, es conveniente hacer uso de tecnología IoT para mejorar el control y monitoreo del sistema de bombeo de servicio de agua en tiempo real, y de esta manera, hacer prácticas y seguras las tareas de mantenimiento. En ese sentido, el objetivo de este trabajo es presentar los resultados del desarrollo de la plataforma integral Ameyali, la cual se conforma de tres módulos principales para el control y monitoreo del sistema de abastecimiento de agua: a) una aplicación móvil para control a distancia del sistema de bombeo mediante conexión vía Bluetooth o SMS. b) un dispositivo electrónico ubicado en la instalación de la bomba, la cual se conecta mediante GPRS o Bluetooth a la aplicación móvil y los sensores para el monitoreo de temperatura, c) una aplicación Web para visualizar los datos de temperatura obtenidos a través de los sensores.

\section{Antecedentes}

Existen trabajos realizados mediante tecnologías e implementación de arquitecturas en distintas partes del continente para combatir problemáticas similares. 
En (Quezada, Bautista, \& Flores, 2014) implementaron un sistema de control y monitoreo de descarga de agua en un pozo de agua potable. En la etapa de diseño crearon las interfaces gráficas de usuario (Graphical User Interface, GUI) para interactuar con el operador y la interfaz hombre-máquina (Human Machine Interface, HMI); ésta última se implementó en software propietario contemplando reglas para el control y monitoreo de las condiciones del sistema. Programaron la HMI interconectado con un controlador lógico programable (Programmable Logic Controller, PLC), obteniendo el control de la maquinaria del pozo.

En (Rodríguez \& García, 2014) automatizaron la planta de tratamiento de agua para uso industrial en Colcerámica S.A. planta Girardota, obtuvieron un diagnóstico para establecer las variables críticas: $\mathrm{pH}$, turbidez, flujo y nivel de agua en los vertederos; realizaron los diagramas de Tubería e Instrumentación (P \& ID) y el plano 3D de la planta automatizada y formularon los cálculos costo-beneficio generados al implementar la propuesta. Como resultados, la medición exacta de las variables involucradas, registro continuo de las mismas, exceptuando la variable $\mathrm{pH}$; un sistema de dosificación automático para el sulfato de aluminio e hipoclorito de sodio, para que el agua tratada en la planta cumpla con las condiciones requeridas por la compañía.

(Pérez, Koo, García, \& Carmona, 2014) proponen un sistema de riego agrícola automatizado, monitoreado y controlado remotamente desde cualquier parte del mundo donde exista servicio de telefonía celular. Buscaron productos de bajo costo para utilizarlos y brindar al usuario un servicio accesible. Las principales herramientas que utilizaron son: Arduino para la automatización del sistema y Android para la interacción con el usuario. Como resultado ofrecen un monitoreo de datos y control del sistema de riego a través de internet, logrando seguridad y flexibilidad al agricultor.

(Robayo, Silva, \& Mosquera, 2015) proporcionan un sistema de control computarizado para automatizar la planta "La Esmeralda" que realiza el cargue de agua potable para camiones cisterna. El sistema tiene instrumentos electrónicos que controlan las bombas y válvulas del tanque de almacenamiento, realiza el arreglo necesario para ubicar y montar el sensor ultrasónico para monitorizar la apertura y cierre de las válvulas, el encendido y apagado de las bombas sumergibles y el nivel del tanque en tiempo real. La planta ofrece suministro automático de los volúmenes de agua requeridos por el cliente, todo controlado por medio de una aplicación en LabView que monitorea y visualiza en tiempo real los acontecimientos que genere el proceso de cargue de agua potable a los camiones cisterna.

(Chueca, 2017) reportaron un sistema automatizado para la gestión de una planta de tratamiento de aguas residuales. El control se realizó a través de un PLC y el manejo de la planta automatizada así como la visualización de todas las condiciones que la atañen, se realizó a través de una pantalla HMI conectada al PLC. Los registros de los procesos internos se van creando en un servidor FTP para su análisis posterior. El usuario puede visualizarlos y contrastarlos.

R. López implementó una interfaz SCADA para la visualización en tiempo real del sistema de distribución de agua potable de la ESSAP en la ciudad de Coronel Oviedo. El proyecto implementa sensores de nivel de agua, sensores de presión, servomecanismos para controlar válvulas principales y las diferentes señalizaciones asociadas a un control 
central, para monitoreo constante de todo el proceso de manera automática. El lenguaje utilizado fue JAVA y el microcontrolador fue Arduino Uno (López-Benítez, 2017).

F. A. Calle y J. X. Gaibor, propusieron un sistema de riego automático para dispositivo móvil, el usuario envía un mensaje de texto hacia la Shield GPRS/GSM SIM9oo, el texto es leído por la tarjeta Arduino y acciona los pulsadores para activar la PLC, de esa manera se enciende el riego por goteo en las plantaciones de ASOFRUT ubicada en la ciudad de Ambato (Calle-Zambrano, 2017).

Calderón et al. desarrollaron un prototipo con componentes electrónicos compatibles con el paradigma de IoT (Internet of Things, Internet de las Cosas) para monitorear el nivel de agua y el llenado de contenedores. Se hace la lectura del sensor de nivel por parte del Arduino, cada 5 segundos; se identifica la diferencia clasificándose de nivel alto, normal o bajo, se envía el dato al módulo transmisor y lo dirige hacia la estación central. La tecnología se aplicó en una planta de tratamiento de agua, y mantuvieron en constante monitoreo por 24 horas cada 6 segundos. La aportación principal es el uso de sensores de nivel de agua (Calderón, Junio 2018).

O. J. López aportó un sistema automático para monitorear y accionar la bomba de agua, mediante comunicación inalámbrica implementando una infraestructura de red basada en una tecnología Wifi con un estándar 802.11, para controlar el encendido y apagado del bombeo, y detectar el nivel de agua existente con sensores en una interfaz gráfica desarrollado en LabView, el beneficio es para la comunidad de La Parroquia, Pimocha (López-Correa, 2018).

M. A. Marueta diseñó e implementó el prototipo de un sistema SCADA basado en un microcontrolador para control del tren de filtración (tratamiento primario) de una planta de depuración de aguas grises. la finalidad fue proveer un control flexible del sistema a través de Internet por los volúmenes de agua que se manejan. El sistema alojado en una $\mathrm{PC}$, permite que el microcontrolador ejecute el algoritmo de control del proceso, con las funciones de SCADA (Murueta, 2019).

\section{Aplicación de la metodología}

Para el desarrollo de la plataforma integral, se implementó el Modelo Orientado a Prototipos representado en la Figura 1.

Las etapas que conforman el Modelo Orientado a Prototipos son: investigación preliminar; especificación de requerimientos y prototipo; diseño técnico; programación y pruebas; operación y mantenimiento. Durante la etapa de investigación preliminar se definió la problemática y se establecieron los aspectos y la factibilidad del desarrollo del producto software.

La etapa de especificación de requerimientos y prototipo se compone de 4 fases: "Análisis”, "Diseño y construcción”, "Evaluación” y "Modificación”. En la primera fase "Análisis" para el análisis de requerimientos se utilizó la plantilla estándar IEEE83o (IEEE, std 830-1998), se generó la carta del proyecto (Project Charter) y un plan de gestión del proyecto basado en el PMBOK Sexta Edición. En la segunda fase de "Diseño y construcción”, con base en los requerimientos funcionales, se realizó el diseño de los 
diagramas de casos de uso, secuencia, actividades y componentes. También se diseñó un prototipo de las interfaces gráficas de usuario para visualizar la navegabilidad de la aplicación Web y móvil.

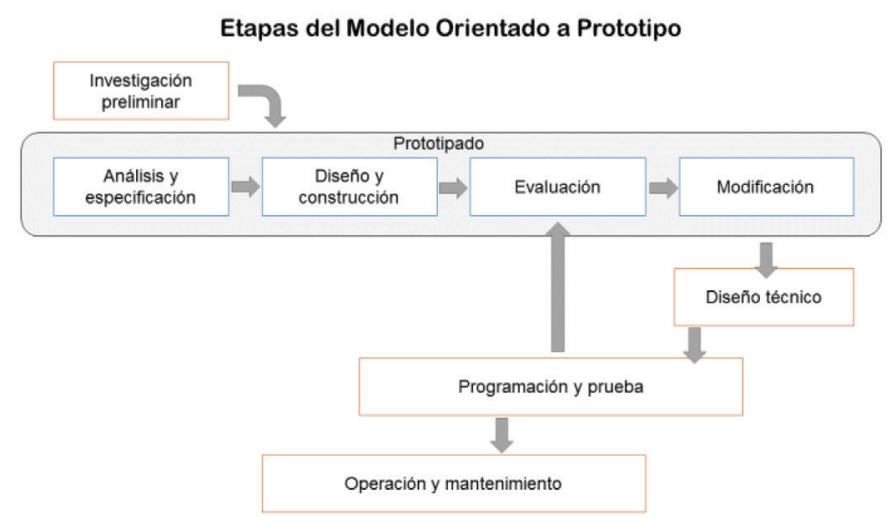

Figura 1 - Las etapas del Modelo Orientado a Prototipos

Durante la fase de "Evaluación" se realizaron reuniones con el cliente para validar el diseño, la funcionalidad y los futuros entregables del proyecto. Después de evaluar el prototipo de interfaces, él cliente realizó observaciones y comentarios como parte de la mejora del prototipo inicial. Con base en los comentarios y observaciones realizadas por el cliente, en la fase de "Modificación" se realizaron cambios en el diseño del prototipo inicial, así como ajustes en los requerimientos funcionales, por lo cual se modificó el contenido de la plantilla IEEE830. En la siguiente etapa denominada programación y pruebas, se construyó cada uno de los módulos que componen las diversas partes de la plataforma integral.

En la etapa de pruebas de funcionalidad, se realizaron pruebas de integración, es decir, durante la codificación, cada componente (electrónico y software) fue probado de manera individual validando los datos de entrada y salida. Por último, la etapa de operación y mantenimiento permitió la implementación de la plataforma integral en un ambiente de producción. Este ambiente de producción se divide en tres partes: el primero, un servidor Web, donde se hospeda la aplicación Web de manera local, para esto, en la oficina de la agencia municipal de la comunidad de Huixtitla; el segundo ambiente, es un dispositivo móvil con sistema operativo Android, en este dispositivo se instaló la aplicación móvil para el control remoto de la bomba de agua; por último, el tercer ambiente, es el panel de control eléctrico, ubicado en las instalaciones del sistema de bombeo. Aquí se realizó la adaptación del cableado eléctrico del encendido de la bomba, agregando el módulo de control para operar la bomba de manera remota. 


\section{Resultados}

Los resultados obtenidos en la primera etapa de la metodología fue información preliminar de la instalación del sistema de bombeo: a) el tiempo de traslado existente entre la instalación de la bomba y la comunidad de Huixtitla es de 40 minutos a pie, b) una persona es encargada de encender y apagar la bomba por ello recorre el camino dos veces al día (mañana y tarde), c) el encendido de la bomba usa un circuito de corte, por ello brinda la posibilidad de realizar configuraciones en el control, d) la instalación eléctrica permite la conexión de otras herramientas y aparatos.

La segunda etapa "Especificación de requerimientos y prototipos" se conforma por 4 fases: "Análisis y especificación", "Diseño y construcción", "Evaluación” y "Modificación". En la etapa "Análisis y especificación" se realizó la recopilación de datos para identificar los tipos de usuarios y obtener los requerimientos específicos mediante el estándar IEEE830. A continuación, se mencionan algunos requerimientos funcionales.

- Tener acceso al funcionamiento de la bomba a distancia

- Encender la bomba mediante el uso del teléfono celular y a larga distancia

- Apagar la bomba mediante el uso del teléfono celular y a larga distancia

- Encender la bomba mediante el teléfono celular a una corta distancia

- Apagar la bomba mediante el teléfono celular a una corta distancia

- Encender y apagar el sistema de bombeo de manera manual

- Monitorear el funcionamiento del sistema de bombeo

La etapa "Diseño y construcción" implementa diagramas UML para establecer el diseño y funcionamiento de la arquitectura de la plataforma integral. En la figura 2 se presenta el diagrama de casos de uso de la aplicación móvil. En este caso de uso se definió el rol Operador, el cual representa a la persona que utilizará la aplicación móvil. De la misma manera, se definieron los casos de uso que modelan las principales funciones de la aplicación: encender vía SMS, este caso de uso permite al usuario enviar un SMS desde la App móvil para indicar el encendido de la bomba; apagar vía SMS, mediante este caso de uso, el usuario puede indicar el apagado de la bomba; encender vía Bluetooth, como alternativa a los dos casos anteriores, la aplicación permite encender el sistema de bombeo mediante Bluetooth; y por último, el caso de uso apagar vía Bluetooth, realizando la función contraria al caso anterior vía Bluetooth.

Para representar la modularidad de la plataforma integral, las dependencias entre ellas, la comunicación y el medio de enlace se presenta el diagrama de componentes donde se observan 5 paquetes (ver Figura 3), el paquete "Dispositivo móvil" se comunica con el paquete "Red móvil" con los módulos que controlan el funcionamiento de la bomba mediante señal GPRS, a su vez los comandos AT son recibidos por el módulo SIM9oo que transmite la orden de encendido o apagado a la placa ARDUINO para activar los sensores de temperatura. 


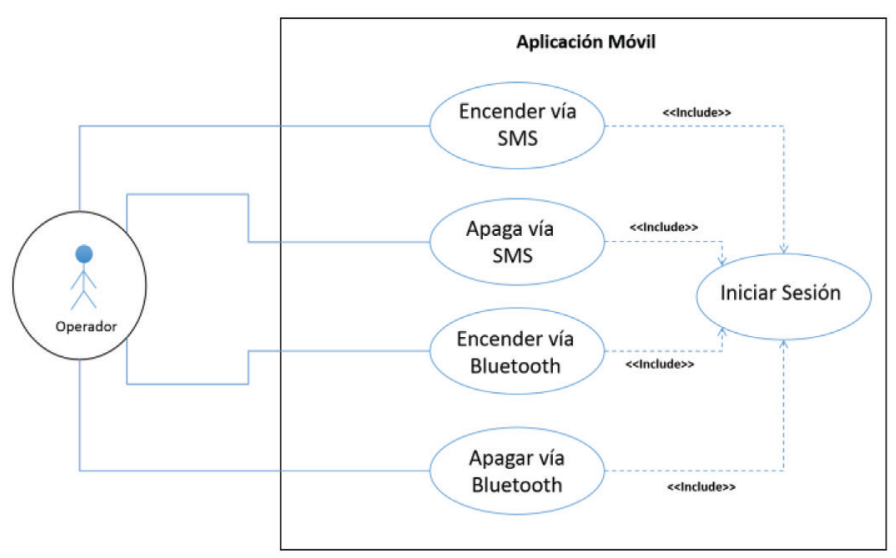

Figura 2 - Diagrama de casos de uso de la aplicación móvil

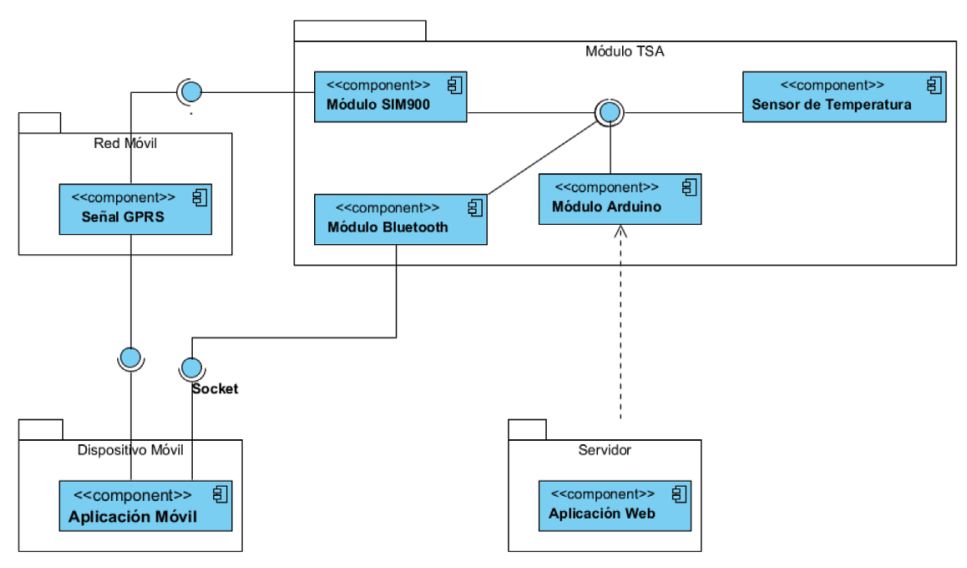

Figura 3 - Diagrama de componentes principales de la plataforma

Con base en el modelo $4+1$ vistas, propuesto por Philippe Kruchten (Kruchten, 1995), se diseñó la arquitectura de software de la plataforma AMEYALI, en donde se establecen cada uno de los componentes y la comunicación para el funcionamiento de toda la plataforma integral. En la Figura 4, se visualiza cada uno de los elementos que forman parte de la arquitectura, así como la interacción entre ellos. 


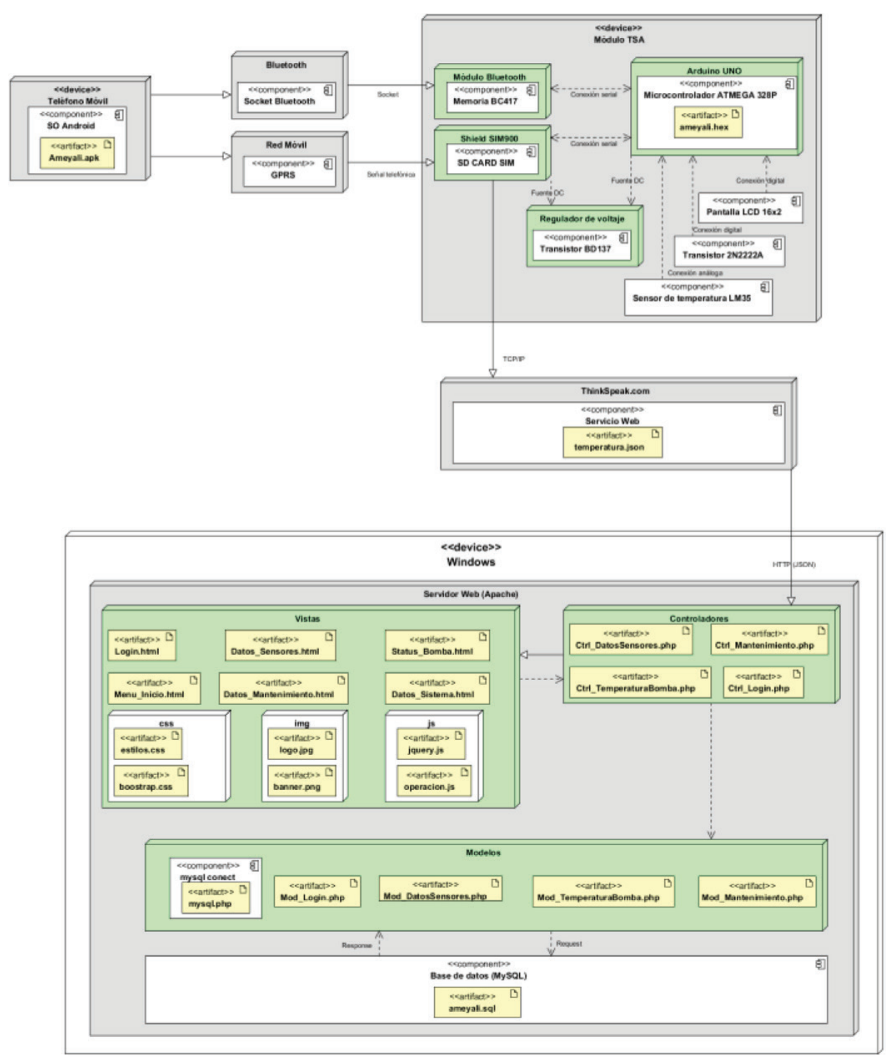

Figura 4 - Arquitectura de la plataforma (vista física)

Cada uno de los nodos tiene una funcionalidad esencial para la comunicación, en la tabla 1 , se describen los más importantes.

\begin{tabular}{|c|c|}
\hline Nombre & Documentación \\
\hline Módulo TSA & $\begin{array}{l}\text { El módulo TSA es una caja con dimensiones de } 21 \times 21 \times 8 \mathrm{~cm} \text {. elaborado con material } \\
\text { de plástico para evitar descargas eléctricas hacia el operador. Dentro de ella contiene } \\
\text { dispositivos electrónicos, cables de conexión, módulos de relevadores, módulos de } \\
\text { comunicación y una conexión de corriente hacia la bomba, para hacerlo funcionar a } \\
\text { distancia y de manera directa. Contiene un sensor de temperatura que va a monitorear } \\
\text { la bomba en tiempo real, para enviar los datos hacia la aplicación Web. }\end{array}$ \\
\hline Arduino UNO & $\begin{array}{l}\text { Este microcontrolador funciona con un voltaje de } 5 \mathrm{~V} \text { a } 2 \mathrm{~A} \text {, por ello es necesario } \\
\text { equilibrar la energía que se le conecta. Debe tener cargado el código de programación } \\
\text { ameyali.hex, dentro del código ya se especifican las entradas de los demás dispositivos, } \\
\text { y el orden en que los hará funcionar. Controla la entrada de datos (Bluetooth y } \\
\text { SIM90o) y el monitoreo del sensor. }\end{array}$ \\
\hline
\end{tabular}




\begin{tabular}{|c|c|}
\hline Nombre & Documentación \\
\hline $\begin{array}{l}\text { Módulo } \\
\text { Bluetooth }\end{array}$ & $\begin{array}{l}\text { Contiene las configuraciones dentro de la memoria } \mathrm{BC} 417 \text { para ejercer una conexión } \\
\text { segura y privada hacia la aplicación móvil, los comandos para su operación son las } \\
\text { siguientes: } \\
\text { 1. Nombre: Ameyali_CCA_01 } \\
\text { 2. SSID: } 0808 \\
\text { 3. Velocidad en Baudios: } 19200\end{array}$ \\
\hline Teléfono Móvil & $\begin{array}{l}\text { Cualquier marca de teléfono móvil que cumpla con las siguientes características: } \\
\text { - Contar con servicios GPRS } \\
\text { - Tener acceso a conectividad vía Bluetooth } \\
\text { - Tener una versión de Android 4.o en adelante } \\
\text { - Instalar la aplicación ameyali.apk } \\
\text { - Abrir aplicación para comprobar la función que realiza }\end{array}$ \\
\hline Shield SIM9oo & $\begin{array}{l}\text { Se debe considerar que este módulo debe contener un Chip de la compañía Telcel, } \\
\text { ya que las configuraciones se realizaron para ese fin. El voltaje que necesita es de } \\
5 \mathrm{~V} \text { a } 3 \text { A. }\end{array}$ \\
\hline $\begin{array}{l}\text { Sensor de } \\
\text { temperatura } \\
\text { LM35 }\end{array}$ & $\begin{array}{l}\text { Se conecta cerca de la bomba de agua, para monitorear constantemente la } \\
\text { temperatura y enviar los datos hacia la tarjeta de Arduino. }\end{array}$ \\
\hline Vistas & $\begin{array}{l}\text { La carpeta vistas contiene los siguientes tipos de archivos los cuales se comunican } \\
\text { entre sí para mostrar las visualizaciones al usuario: } \\
\text { - Clases, éstas tienen la tarea de darle forma al contenido, para que el usuario pueda } \\
\text { realizar las tareas que necesita. } \\
\text { - JS, estos archivos realizan acceso a datos y manejan operaciones de consulta hacia } \\
\text { el servicio en Thingspeak } \\
\text { - CSS, brinda diseño a las ventanas, ejemplo de ello son los colores, figuras y formas. } \\
\text { - Img, archivos que complementan las ventanas, imágenes, videos y sonidos. }\end{array}$ \\
\hline Controladores & $\begin{array}{l}\text { La programación de clases se hizo en la arquitectura MVC (Modelo Vista y } \\
\text { Controlador). Los controladores tienen la función de administrar las operaciones hacia } \\
\text { los modelos, esto mediante la programación Orientada a Objetos. }\end{array}$ \\
\hline Modelos & $\begin{array}{l}\text { Los modelos son archivos con código funcional que ejecuta operaciones hacia el gestor } \\
\text { de base de datos, consultar, modificar, insertar, y en su caso eliminar. }\end{array}$ \\
\hline
\end{tabular}

Tabla 1 - Descripción de los componentes en la arquitectura general

Los diagramas que dan soporte a la arquitectura de la plataforma integral se desarrollaron en la etapa de "Diseño técnico". Los diagramas obtenidos son de clases, esquemáticos (donde se modela el módulo electrónico de comunicación), diagrama de objetos y diagramas PCB. Estos diagramas se diseñaron con las herramientas CASE Visual Paradigm Enterprise v10.0 y Autodesk Eagle v8.2.1.

Para el desarrollo del módulo de control, se diseñó un diagrama esquemático para modelar la comunicación entre los componentes electrónicos. Estos componentes electrónicos son: placa Arduino R3, Shield SIM90o, pantalla LCD 2x16, módulo Bluetooth, LEDs, resistencias, transistores, sensor de temperatura LM35, relay. En la Figura 5A se visualiza el diagrama esquemático del módulo de control, en la figura $5 \mathrm{~B}$ se visualiza el módulo TSA que se desarrolló siguiendo el diagrama esquemático. 


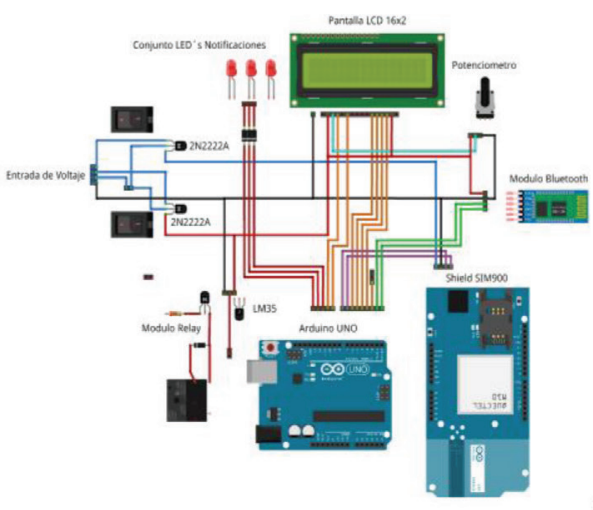

Figura 5A - Diagrama esquemático del módulo de control

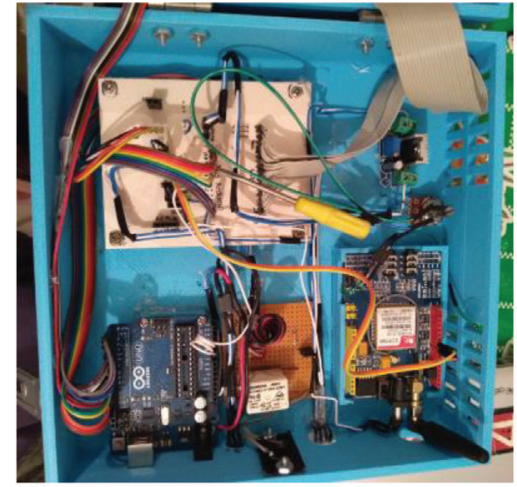

Figura 5B - Módulo TSA

\section{Evaluación de usabilidad de la plataforma integral}

Kitchenham (Kitchenham, 1997) propuso una metodología para la evaluación de métodos y características cuantitativas y cualitativas en ingeniería del software. Kitchenham afirma que una evaluación cualitativa es ampliamente recomendada para una evaluación de usabilidad. Por su parte, Brooke y Sauro proponen el System Usability Scale que es una herramienta rápida y confiable para medir la usabilidad (J., 1996) (Jeff, 2011).

En la evaluación de la usabilidad de plataforma integral para el control y monitoreo del sistema de abastecimiento de agua en la comunidad de Huixtitla se implementó el System Usability Scale que es una herramienta metodológica muy similar a la Escala de Likert y que se usa para medir la usabilidad de un objeto, dispositivo, aplicación o plataforma; es una manera rápida y confiable de medir la usabilidad.

\section{Diseño de estudio}

Para la evaluación cualitativa de usabilidad de la plataforma integral para el control y monitoreo del sistema de abastecimiento de agua en la comunidad de Huixtitla se implementó un cuestionario con escala System Usability Scale (SUS) para evaluar aspectos como la facilidad de uso, experiencia de seguridad y experiencia de uso; para ello se elaboraron las siguientes preguntas, en las cuales el usuario tuvo que responder si estaba fuertemente en desacuerdo, fuertemente de acuerdo o alguna respuesta intermedia.

1. ¿'Me gustó usar los componentes de la plataforma integral?

2. ¿Me sentí muy seguro usando la plataforma?

3. ¿Considero que la plataforma es fácil de usar? 
4. ¿Considero que no necesito apoyo de una persona técnica para usar los componentes de la plataforma?

5. ¿No necesito aprender muchas cosas antes de comenzar a utilizar la plataforma?

6. ¿No necesito configurar muchas cosas antes de utilizar la plataforma?

7. ¿Considero que una persona aprendería a usar la plataforma muy rápidamente?

8. ¿Considero que es sencillo utilizar la aplicación móvil para control a distancia del sistema de bombeo mediante conexión vía Bluetooth o SMS?

9. ¿Considero que es fácil de utilizar el dispositivo electrónico ubicado en la instalación de la bomba?

10. ¿Considero que es sencillo de utilizar la aplicación Web para visualizar los datos de temperatura obtenidos a través de los sensores?

\section{Participantes}

La evaluación de usabilidad está orientada a la persona responsable del sistema de bombeo del agua en la comunidad de Huixtitla cuya responsabilidad es encender y apagar diariamente el sistema, además de monitorear su funcionamiento y realizar el mantenimiento; así como a personas que conocen o realizaron tareas relacionadas con el sistema de bombeo del agua de la comunidad. El objetivo de la evaluación de usabilidad es comprobar que cualquier persona sin habilidades de programación y sin conocimientos de aplicaciones Web, aplicaciones móviles o Internet de las cosas implementen la plataforma integral para el control y monitoreo del sistema de abastecimiento de agua de manera fácil, segura y rápida. Cabe mencionar los participantes no tenían conocimiento del System Usability Scale por lo que se les dio una introducción del SUS y del instrumento empleado para la evaluación.

\section{Procedimiento de recopilación de datos}

Para la evaluación de usabilidad se les pidió a los participantes que usarán todos los componentes de la plataforma integral de la manera que lo harían en un día normal de trabajo; posteriormente a su uso se aplicó a los participantes un cuestionario basado en la escala de SUS para evaluar la usabilidad de la herramienta, el cuestionario evaluó aspectos como facilidad de uso, entre otros aspectos con una escala de respuesta del 1 al 5 que equivale del "Totalmente en desacuerdo" al "Totalmente de acuerdo", los participantes tuvieron un lapso de 5 minutos para contestar el cuestionario.

\section{Resultados de la evaluación de usabilidad}

En esta sección se presentan los resultados de la evaluación de usabilidad de la plataforma integral para el control y monitoreo del sistema de abastecimiento de agua en la comunidad de Huixtitla utilizando la escala de SUS. Los resultados obtenidos del cuestionario de evaluación de usabilidad que se representan en la tabla 2 corresponden a la media aritmética de las respuestas de los participantes, una representación de los resultados se presenta en la Figura 6. 


\begin{tabular}{|c|c|}
\hline Pregunta & Media \\
\hline ¿Me gustó usar los componentes de la plataforma integral? & 4.8 \\
\hline ¿Me sentí muy seguro usando la plataforma? & 4.1 \\
\hline ¿Considero que la plataforma es fácil de usar? & 4.4 \\
\hline $\begin{array}{l}\text { ¿Considero que no necesito apoyo de una persona técnica para usar los componentes de la } \\
\text { plataforma? }\end{array}$ & 3.6 \\
\hline ¿No necesito aprender muchas cosas antes de comenzar a utilizar la plataforma? & 3.6 \\
\hline ¿No necesito configurar muchas cosas antes de utilizar la plataforma? & 3.4 \\
\hline ¿Considero que una persona aprendería a usar la plataforma muy rápidamente? & 4.5 \\
\hline $\begin{array}{l}\text { ¿Considero que es sencillo utilizar la aplicación móvil para control a distancia del sistema de } \\
\text { bombeo mediante conexión vía Bluetooth o SMS? }\end{array}$ & 4.7 \\
\hline $\begin{array}{l}\text { ¿Considero que es fácil de utilizar el dispositivo electrónico ubicado en la instalación de la } \\
\text { bomba? }\end{array}$ & 4.8 \\
\hline $\begin{array}{l}\text { ¿Considero que es sencillo de utilizar la aplicación Web para visualizar los datos de } \\
\text { temperatura obtenidos a través de los sensores? }\end{array}$ & $4 \cdot 3$ \\
\hline
\end{tabular}

Tabla 2 - Resultados de la evaluación de usabilidad

Los resultados obtenidos en la evaluación de usabilidad fueron favorables como se puede observar en la tabla 2, la media aritmética en la mayoría de las preguntas mayor o igual a 4 de 5 que representa "Totalmente de acuerdo" con un $70 \%$ de las preguntas y un 30\% de las preguntas tendientes a 3.6 que representa "Normal"; lo que representa una evaluación de usabilidad satisfactoria en la cual los participantes estuvieron de acuerdo en todas las preguntas. Los resultados del SUS en el uso de la herramienta, la media de las puntuaciones arroja un resultado favorable "de acuerdo" (4.22) con una pequeña desviación estándar (o.1878) lo que implica que los participantes tuvieron una experiencia de usabilidad favorable con respecto a su interacción con la plataforma integral para el control y monitoreo del sistema de abastecimiento de agua en la comunidad de Huixtitla.

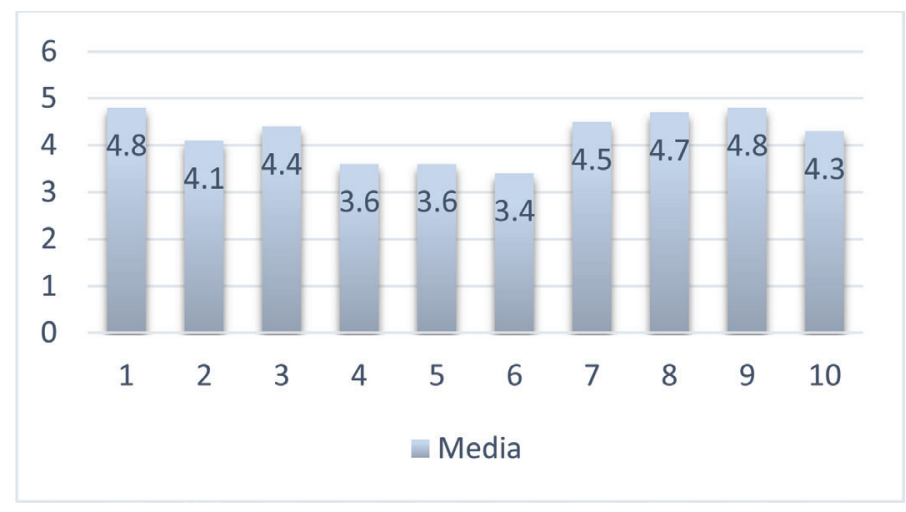

Figura 6 - Representación gráfica de la evaluación de usabilidad 
Las preguntas que obtuvieron un mejor puntaje fueron: ¿̇me gustó usar los componentes de la plataforma integral? Y ¿ंconsidero que es fácil utilizar el dispositivo electrónico ubicado en la instalación de la bomba? La razón es porque estos elementos contienen una interfaz intuitiva para el usuario, las medidas cómodas para instalar y operar.

La evaluación realizada por los habitantes arroja que la plataforma integral es fácil de usar, no se necesita tener un conocimiento amplio de las tecnologías, cualquier persona que sepa leer y escribir puede aprender a usar los componentes, la aplicación móvil y Web son sencillos de operar, los colores y espacios dentro de la plataforma les gustó a los usuarios. Estas características fueron aceptadas y aprobadas gracias a la etapa de evaluación y modificación en la que el cliente dio precisiones acerca de las interfaces gráficas.

Las preguntas con menor puntaje fueron: ¿no necesito configurar muchas cosas antes de utilizar la plataforma? Y ¿̇no necesito aprender muchas cosas antes de comenzar a utilizar la plataforma? Con valoración de "Normal", esto de acuerdo a los comentarios que dieron los usuarios, ya que recientemente están trabajando con una computadora y con un sistema así, necesitaron de una capacitación inicial pero con la práctica diaria mejoraron sus conocimientos.

Dentro de las 10 preguntas, se consideran 2 como las más importantes ya que esto determina las decisiones a futuro de la plataforma, cada uno tuvo diferentes porcentajes en los resultados de la evaluación. La figura 7A representa la facilidad de uso de la aplicación móvil para controlar el sistema de bombeo, el 70\% está totalmente de acuerdo ya que contiene interfaz intuitivo y la operatividad es práctica.

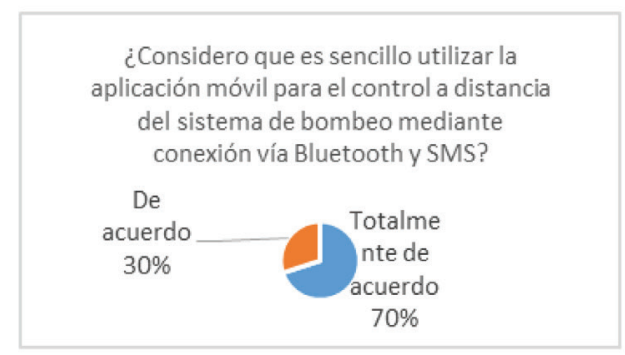

Figura $7 \mathrm{~A}$ - Gráfica representativa de la facilidad de uso de la aplicación móvil para controlar el sistema de bombeo

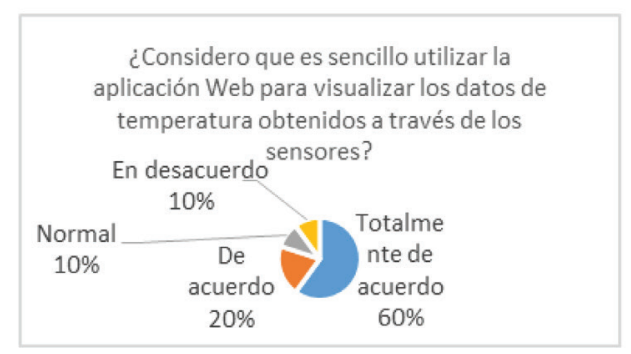

Figura 7B - Gráfica representativa de la facilidad de uso de la aplicación Web para visualizar los datos de la temperatura obtenidos a través de los sensores

La Figura 7B muestra la gráfica acerca del uso de la aplicación Web para visualizar los datos que monitorean los sensores. El 60\% está totalmente de acuerdo, pero también se puede notar que hay un 10\% en desacuerdo y normal, lo que con llevó analizar la razón, una vez conversando con los usuarios comentaron que no todos saben manejar una 
computadora, seleccionar datos en la gráfica para mayor información y que necesitan de mayor familiarización.

\section{Conclusiones}

La plataforma integral para el control y monitoreo del sistema de abastecimiento de agua en la comunidad de Huixtitla cumple como una solución tecnológica a cada una de las necesidades especificadas por el cliente, gracias a la implementación de Internet de las cosas.

El trabajo realizado define la manera en que dos tipos de aplicaciones en ambientes Web y móvil interactúan con dispositivos electrónicos como la placa Arduino Uno y sensores de temperatura para controlar el encendido y apagado de una bomba de agua, donde se implementó servicios Web alojados en la plataforma ThingSpeakTM.

\section{Beneficios obtenidos}

Durante las pruebas funcionales se obtuvo la conexión estable de los dispositivos, el envío de datos y la visualización de información de manera directa, ahorrando tiempo y trabajo de los usuarios y beneficiando la operatividad del sistema de bombeo.

Los usuarios de las aplicaciones comentaron el gran beneficio que obtuvieron al utilizar la tecnología desarrollada y aplicada en este caso de estudio, ya que realizan el encendido y apagado de forma remota y segura con un solo click sin exponer la integridad de ningún usuario, disponen de un sistema de agua automatizado, práctico e intuitivo que puede ser utilizado en horarios poco adecuados para los operadores del servicio.

Una arquitectura escalable, esta plataforma, además de ayudar a las comunidades rurales, también sirve como un marco de referencia en el desarrollo de aplicaciones basadas en el IoT, ya que la arquitectura diseñada, al ser completamente modular, con un bajo acoplamiento y alta cohesión, permite agregar o quitar funcionalidades para controlar nuevos dispositivos electrónicos y sensores.

\section{Trabajos a futuro}

Como trabajo a futuro se realizará la solicitud de registro de patente de un modelo de utilidad ante el Instituto Mexicano de la Propiedad Intelectual.

Este trabajo apoyará a futuros proyectos e investigaciones (como seguridad y análisis de riesgos, predicción de mantenimientos y daños mediante técnicas de IA aplicadas a la domótica, entre otras) referentes a la programación en Arduino y Android, también en la conexión de módulos como lo son la Shield SIM90o y Bluetooth mediante la publicación de los resultados en este artículo.

Por otro lado, se pretende escalar la funcionalidad de plataforma para el control y monitoreo de otros servicios para la comunidad, como el alumbrado público, en ese sentido, se pretende llevar a cabo convenios con Ayuntamientos para la implementación de AMEYALI en sus comunidades. 


\section{Agradecimientos}

A los habitantes de la comunidad de Huixtitla, Veracruz por otorgar las facilidades y accesos a las instalaciones donde se ubica el sistema de abastecimiento de agua y permitir el desarrollo de este proyecto.

Al Instituto Tecnológico Superior de Zongolica por el apoyo y los espacios proporcionados para realizar este trabajo.

Al Dr. Emmanuel de Jesús Ramírez Rivera por los conocimientos aportados para la escritura de este artículo.

\section{Referencias}

Android Developers. (2018). Obtenido de https://developer.android.com/ studio/releases/

Calderón, C. A. (2018). Monitoreo y automatización del proceso de bombeo y almacenamiento de agua potable aplicado a una Planta de Tratamiento de Agua. In: 2018 13th Iberian Conference on Information Systems and Technologies (CISTI), pp. 1-6. Caceres: IEEE.

Calle-Zambrano, J. X.-V. (2017). Automatización de un sistema de riego con monitoreo local usando una touch y control remoto inalámbrico vía GSM (Arduino Open Source) para el mejoramiento dentro del campo agrícola en Asofrut de la Ciudad de Ambato. Riobamba: ESPOCH.

Chueca, P. (2017). Automatización de una planta de tratamientos de aguas residuales. Retrieved from: http://hdl.handle.net/10251/88981.

CONAGUA. (2007). Manual de Agua Potable, Alcantarillado y Saneamiento. Coyoacán, D.F., México.

Evans, D. (2011). Internet de las cosas. Cómo la próxima evolución de Internet lo cambia todo. San José, CA.: Cisco IBSG.

Gauchat, J. D. (2012). El gran libro de HTML5, CSS Y Javascript. Barcelona, España: Marcombo.

Ingeniería de software. (2017). Recuperado el 04 de abril de 2018, de: http://ingenieriadesoftware.mex.tl/63758_AUP.html

Brooke, J. (1996). SUS: a quick and dirty, usability scale. London: Taylor and Francis.

Jeff, S. (2011). Measuring usability with the System Usability Scale (SUS). MeasuringU.

Kitchenham, B. B.-P. (1997). Evaluation and assessment in software engineering. Information \& Software Technology. DOI:10.1016/So950-5849(97)00024-4

Kruchten, P. B. (1995). The 4+1 View Model of Architecture (Vol. 12). IEEE Software. 
López-Benítez, R. (2017). Diseño de automatización del sistema de distribución de agua potable de la ESSAP en la ciudad de Coronel Oviedo. Coronel Oviedo: Universal Nacional de Caaguazu.

López-Correa, O. J. (2018). Sistema Automático para el monitoreo de servicios de agua potable de la Parroquia Pimocha del Cantón Babahoyo. Babahoyo: Universidad Técnica de Babahoyo.

Murueta, M. A. (2019). Automatización de planta de tratamiento de aguas con microcontrolador y servicio Web. Cd México: Ciudad Universitaria.

Martin Fomler, K. S. (1999). UML gota a gota. México: Addison Wesley Longman.

Pérez, B., Koo, J. M., García, F., \& Carmona, J. (2014). Automatización, monitoreo y control remoto de un sistema de riego agrícola con código abierto. LACCEI Latin American and Caribbean Conference for Engineering and Technology, (20), 1-9.

Quezada, J. C., Bautista, J., \& Flores, E. (2014). Diseño e implementación de un sistema de control y monitoreo basado en HMI-PLC para un pozo de agua potable. Ingeniería Investigación y Tecnología, XV(1), 1-10.

Robayo, F. I., Silva, D. M., \& Mosquera, D. J. (2015). Sistema de control automatizado en planta de cargue de agua potable para camiones cisterna. Revista Ingeniería y Región, I(13), 1-10.

Rodríguez, Y. A., \& García, L. E. (2014). Propuesta de automatización de una planta de tratamiento de agua para uso Industrial. Revista Politécnica, X(18), 1. 